\title{
Diamond based quantum technologies
}

\author{
Fedor Jelezko ${ }^{1,2, *}$ \\ ${ }^{1}$ Institute of Quantum Optics, Ulm University, Germany \\ ${ }^{2}$ Center for Integrated Quantum Science and Technology (IQ $\left.{ }^{\text {st }}\right)$, Ulm University, Germany
}

\begin{abstract}
Diamond is not only the king gemstone, but also a promising material in quantum technologies. Optically active impurities (colour centers) in diamond show unique coherence properties under ambient conditions. Their quantum state can be readout and manipulated using a combination of single molecule spectroscopy and magnetic resonance techniques. In this talk it will be shown how engineered spins in diamond can be used for creation of non-classical (entangled) quantum states. I will also demonstrate the potential of atomic magnetometers based on single color centers for nanoscale sensing and imaging. New photoelectric detection technique allowing efficient readout of single color centers will be discussed.
\end{abstract}

Electron and nuclear spin resonance is a spectroscopic technique that provides very detailed information on almost every possible substance but also as very insensitive. This may be contrasted to the excellent sensitivity of optical single molecule spectroscopy which is able to demonstrate the detection of single molecules in condensed matter. A recent development is a combination of these techniques making it possible to detect magnetic resonance transitions of single quantum systems. While first experiments were done with aromatic molecules at low temperatures, recently single spin magnetic resonance techniques were also applied to the study of defect centers in diamond allowing coherent single spin control and readout under ambient conditions. This new approach opened a number of interesting applications, such as quantum information processing and communication, secure quantum communication and sensing with nanometer resolution.

A particularly interesting application of diamond based quantum sensing is the detection of nuclear magnetic resonance on nanometer scales, including the detection of individual nuclear spins or small ensembles of external nuclear spins. Single nitrogen vacancy (NV) color centers in diamond currently have sufficient sensitivity for detecting single external nuclear spins and resolve their position within a few angstroms. The ability to bring the sensor close to biomolecules by implantation of single NV centers and attachment of proteins to the surface of diamond enabled the first proof of principle demonstration of proteins labeled by paramagnetic markers and label-free detection of the signal from a single protein. Single-molecule nuclear magnetic resonance (NMR) experiments open the way towards unraveling dynamics and structure of single biomolecules. However, for that purpose, NV magnetometers must reach spectral resolutions comparable to that of conventional solution state NMR. New techniques were proposed for this purpose and

* Corresponding author: fedor.jelezko@uni-ulm.de 
realized recently including technique that employs quantum entanglement. The ability to sense nuclear spins by NV centers also enables the transfer of polarization from optically polarized spins of NV centers to external nuclear spins. Such diamond based techniques for dynamic nuclear spin polarization are very promising for the enhancement of sensitivity of conventional MRI imaging.

Most of mentioned above results obtained so far with diamond centers are based on optical detection of single NV color centers. Recently it was shown that photoelectrical detection of NV centers base on spin selective photoionization can provide robust and efficient access to spin state of individual color centers. 